\title{
Correlated scattering of protons impinging as hydrogen molecules
}

\section{Citation}

Golovchenko, Jene, and Erik Laegsgaard. 1974. “Correlated Scattering of Protons Impinging as Hydrogen Molecules." Physical Review A 9 (3) (March 1): 1215-1219. doi:10.1103/

physreva.9.1215.

\section{Published Version}

doi:10.1103/PhysRevA.9.1215

\section{Permanent link}

http://nrs.harvard.edu/urn-3:HUL.InstRepos:34334607

\section{Terms of Use}

This article was downloaded from Harvard University's DASH repository, and is made available under the terms and conditions applicable to Other Posted Material, as set forth at http:// nrs.harvard.edu/urn-3:HUL.InstRepos:dash.current.terms-of-use\#LAA

\section{Share Your Story}

The Harvard community has made this article openly available.

Please share how this access benefits you. Submit a story.

\section{Accessibility}




\title{
Correlated scattering of protons impinging as hydrogen molecules
}

\author{
Jene Golovchenko* and Erik Laegsgaard \\ Institute of Physics, University of Aarhus, Denmark \\ (Received 20 July 1973)
}

\begin{abstract}
The joint energy and angular-distribution function of protons transmitted through a thin foil when incident as $2-\mathrm{MeV}_{2}{ }^{+}$has been measured. The experimental result is compared with simple calculations and satisfactory agreement is obtained.
\end{abstract}

In the past few years several workers have reported observing manifestations of molecular binding in scattering experiments with incident molecules of hydrogen. Bacher et al. ${ }^{1}$ saw such effects on the $14.2-\mathrm{MeV}{ }^{12} \mathrm{C}(p, p){ }^{12} \mathrm{C}$ resonance. Caywood et $a l .^{2}$ and Eisen et al.$^{3}$ showed modification of channeling backscatter results, while Poizat et al. ${ }^{4}$ observed $\mathrm{H}_{2}{ }^{+}$molecules emerging from a thin foil. Finally, Stotterfoht et al. ${ }^{5} \mathrm{ob}-$ served a difference in the fluorescence yield of the argon $L$ shell with incident protons and $\mathrm{H}_{2}{ }^{+}$.

In recent experiments we have observed protons transmitted through thin self-supporting carbon foils when the incident particles were either $2-\mathrm{MeV} \mathrm{H}_{2}{ }^{+}$or $1-\mathrm{MeV} \mathrm{H}^{+}$ions, with the motivation of measuring physical phenomena depending upon the correlation of proton positions provided by the binding in the initial hydrogen molecular ions. Our preliminary findings show that the energy and angular distributions of such transmitted protons have a strong dependence upon this correlation. Advantage may be taken of these effects to study proton pairs of a given alignment within the solid. Indeed even the individual spatial identities may be determined. With such capabilities, interesting new approaches towards measuring the interaction of atomic systems become available. In particular, we discuss inelastic excitation of the solid and show that effects due to correlation may be expected.

\section{EXPERIMENTAL PROCEDURE}

The experimental setup used mass-analyzed ions provided by the Aarhus University 2-MeV Van de Graaff facility; incident beam energy fluctuations were less than $1 \mathrm{keV}$; the incident particles were collimated to less than $5 \times 10^{-4}$ rad angular full width at half-maximum (FWHM), and the beamspot size on the sample was $\sim 0.1 \mathrm{~mm}$. It will be seen shortly that such fine collimation is essential for these measurements. Directly behind the thincarbon sample, a small electromagnet swept (at $10 \mathrm{~Hz}$ ) the dispersed transmitted protons in a horizontal plane across a $0.1-\mathrm{mm}$ collimator,
$500 \mathrm{~mm}$ behind the sample. In this way, any representative part of the final angular distribution could be selected for further study. This magnet needed only to supply a maximum deflection of $5 \times 10^{-3} \mathrm{rad}$ for our measurements. Those particles transmitted through the final collimator were energy analyzed in the vertical plane by a magnetic spectrometer with a $40-\mathrm{mm}$-long position-sensitive solid-state detector ${ }^{6}$ in its focal plane. The magnet dispersion $(\Delta X=800 \mathrm{~mm} \cdot \Delta E)$ $E)$ and length of the detector allowed the entire energy region of interest to be studied at one time without changing the magnetic field. Energy resolution for $1-\mathrm{MeV}$ protons was $\sim 3.1 \mathrm{keV}$ FWHM. Figure 1 provides a schematic illustration of the apparatus and the electronics of the data-taking equipment.

Thus, if the energy spectrum obtained is synchronized with the sweeping magnet current (which is a ramp in time), then joint energy and angular distributions are obtained. In addition all results are normalized consistently, with beam-current fluctuations being averaged out over the course of the measurement $(\sim 20 \mathrm{~min})$. Those readers familiar with similar kinds of transmission measurements will recognize the important influence normalization problems have in the design of such experiments.

\section{EXPERIMENTAL RESULTS}

Figure 2 (curve 2) shows the angular distribution of all transmitted protons through an approximately $2-\mu \mathrm{g} / \mathrm{cm}^{2}$ carbon foil with an incident beam of $1-\mathrm{MeV} \mathrm{H}^{+}$. The width of this distribution is 1.46 $\times 10^{-3} \mathrm{rad}$ FWHM and is not well represented by a Gaussian. Also shown in Fig. 2 (curve 3 ) is the angular distribution of transmitted protons when $2-\mathrm{MeV} \mathrm{H}_{2}{ }^{+}$is incident. A rather large broadening and flattening out of the multiple scattering distribution is observed. For completeness the measured angular divergence of the incident $1-\mathrm{MeV} \mathrm{H}^{+}$ beam is also shown (curve 1).

Even more dramatically, Fig. 3 shows the more detailed two-dimensionally-displayed joint energy 
and angular distribution of the emergent protons with $2-\mathrm{MeV} \mathrm{H}_{2}{ }^{+}$incident. The angle is measured relative to the incident beam direction. For zero angle the energy distribution shows two distinct peaks situated in a roughly symmetrical way about half the incident beam energy. As the angle of observation increases, the peaks appear to merge at $\sim 2.5 \times 10^{-3}$ rad. The angular distribution shows a similar behavior as the energy of observation is changed.

\section{DISCUSSION AND ANALYSIS}

The main features of the angular-energy distributions for incident $\mathrm{H}_{2}^{+}$may be understood in terms of simple mechanical arguments. As the incident molecule passes through the first few atomic layers of the foil, the electron responsible for the binding is lost by ionization. The two protons then repel one another via a (screened) Coulomb potential. In the two-proton center-ofmass system, $13.6 \mathrm{eV}$ of potential energy transforms into kinetic energy. (An initial proton separation of $1.06 \AA$ is assumed.? ) This transformation alone results in significant modification of the energy and angular distribution of the beam. For example the proton kinetic energy in the laboratory system, after separation is complete, is

$$
E_{f}=\frac{1}{2} E_{\mathrm{inc}}+\frac{1}{2} E_{p} \pm\left(E_{\mathrm{inc}} E_{p}-E_{\mathrm{inc}}^{2} \sin ^{2} \phi\right)^{1 / 2} \text {. }
$$

Here, $E_{\text {inc }}$ is the incident kinetic energy of the molecule, $E_{p}$ the initial potential energy of the

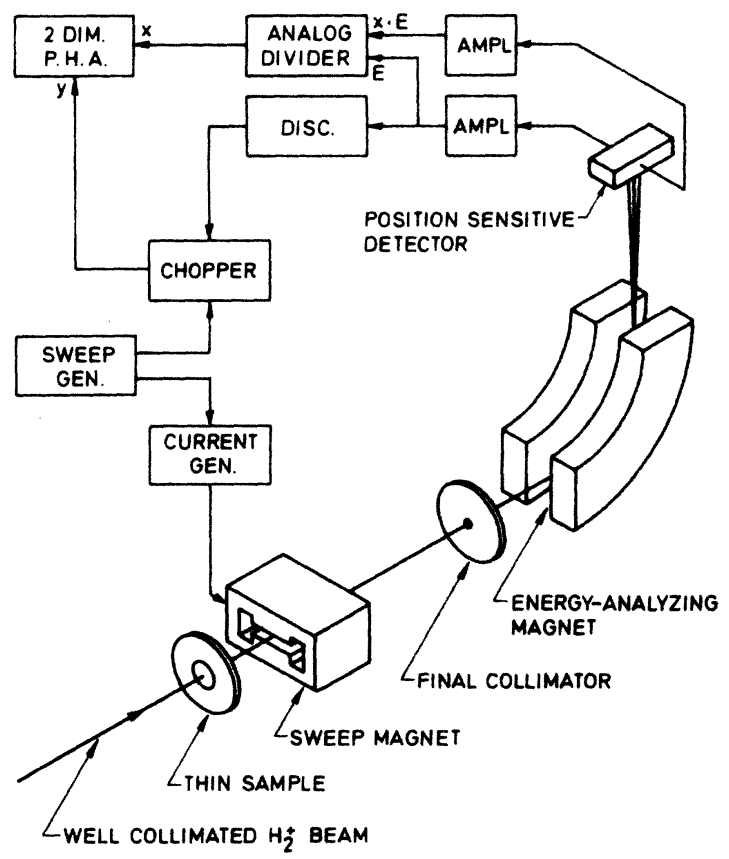

FIG. 1. Schematic illustration of the experiment. two-proton system and $\phi$ is the angle of observation in the laboratory relative to the incident beam direction. (For the interested reader the mathematical details of this problem may be found in the Appendix.) It may be noted that just upon leaving the foil, the proton separation has increased by $\sim 10 \%$ over the initial value. Thus most of the repulsion occurs after the foil.

For our experiments, with 2- $\mathrm{MeV} \mathrm{H}_{2}{ }^{+}$at $\phi=0$, we expect to detect protons of energy 1.0052 and $0.9948 \mathrm{MeV}$ corresponding to protons that break up aligned with the beam direction. (We neglect the small energy loss due to excitation of electrons in the foil.) The higher-energy proton comes from the front position and the lower-energy proton from the back. As the angle $\phi$ is increased, these two energies merge towards one another until finally they meet at $1.000 \mathrm{MeV}$, corresponding to protons breaking up perpendicular to the incident beam direction. Here

$$
\phi=\phi_{\max }=\left(E_{p} / E_{\text {inc }}\right)^{1 / 2} \text {. }
$$

Also assuming the molecular orientation to be isotropic, we find, for the probability per unit solid angle for detecting a proton with $\phi \leqslant \phi_{\max } \ll 1$,

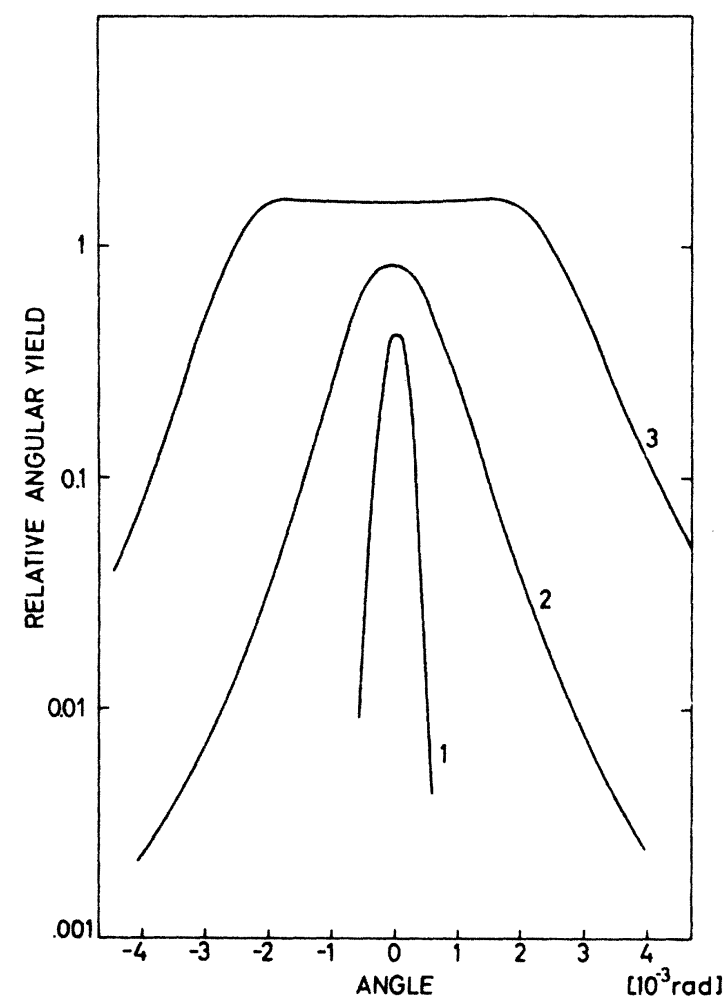

FIG. 2. (1) Angular distribution of the incident beam. (2) Angular distribution of the $\mathrm{H}^{+}$beam after passing the foil. (3) $\mathrm{H}_{2}^{+}$angular distribution after passing through the foil. 


$$
f(\phi)=\frac{1}{2 \pi} \frac{E_{\text {inc }}}{E_{p}}\left(1-\frac{E_{\text {inc }}}{E_{p}} \phi^{2}\right)^{-1 / 2} .
$$

But, alas, all is not so simple. Deviations from the ideal behavior already discussed may result from three causes. Most interesting in these measurements, although least important, is the modification caused by uncertainty in relative position between protons associated with the quantum-mechanical description of the $\mathrm{H}_{2}{ }^{+}$molecule. For an incident particle in the ground state, this effect produces about a $600-\mathrm{eV}$ spread in the energy spectrum obtained at $\phi=0$. (The uncertainty in momentum produces a much smaller effect.) Multiple scattering in the foil and experimental energy resolution completely overwhelm this effect. We have calculated the energy and angular distributions (details are in the Appendix) including multiple scattering and energy resolution as measured from the $\mathrm{H}^{+}$incident case. Figure 4 shows the relative contribution from these effects to the $\phi=0$ case. The agreement $w$ ith the experimental points is deemed quite acceptable. Note should be taken of the fact that the $\mathrm{H}^{+}$multiple scattering distribution is not of a Gaussian type. This is to be expected for such thin foils. The distribution can be represented quite accurately for angles up to $\phi_{\max }$ by a sum of two Gaussians, one having a FWHM of $1.47 \times 10^{-3} \mathrm{rad}$ and a weight of $\frac{4}{5}$, the other having twice this width and a weight of $\frac{1}{5}$. This distribution was used in obtaining the result in Fig. 4. We are not aware of any theoretical estimates that may be compared with this distribution (in this experiment Bohr's parameter $\kappa=Z_{1} Z_{2} e^{2} / h v \approx 1$ ).

Finally, an interesting, though somewhat speculative, application of the above technique may be to study the effect of proton correlation on the inelastic excitation of the solid. It is well known that perturbation treatments of energy loss of fast particles in matter show a proportional dependence on the square of the charge of the penetrating particle. Thus, as the separation between two protons varies from zero to some large value, the energy-loss rate per proton may be expected to change by a factor of 2. A convenient, though perhaps crude, model for estimating the detailed dependence on proton separation and orientation is that of particles penetrating through a freeelectron gas. Calculations in the linear dielectricresponse approximation indicate that, for some orientations and a particle separation of two Bohr radii, one may expect from distant collisions alone a $50 \%$ increase in the average energy-loss rate per particle, compared with only a single penetrating proton. Details of these calculations will be reported later.

Experimental attempts to verify this effect in a quantitative manner have been frustrated by the small energy loss observed in the measurements $(\sim 500 \mathrm{eV})$. We hope to be able to clear up this matter in the not too distant future.

Deepest appreciation for guidance and criticism during the course of this work is due J. U.

Andersen, J. Lindhard, and H. H. Andersen. One of us (JG) would like to express his thanks to the Aarhus University, Institute of Physics, for the privilege and pleasure of being a guest.

\section{APPENDIX}

Consider a fast incident $\mathrm{H}_{2}^{+}$molecule. Neglecting the contribution from rotations and vibrations, the incident kinetic energy is

$$
E_{\text {inc }}=M_{p} v_{\text {inc }}^{2},
$$

where $v_{\text {inc }}$ is the incident proton velocity and $M_{p}$ the proton mass. Assuming that the binding electron is ionized and no longer effects the proton, motions of repulsion occur, resulting in a final

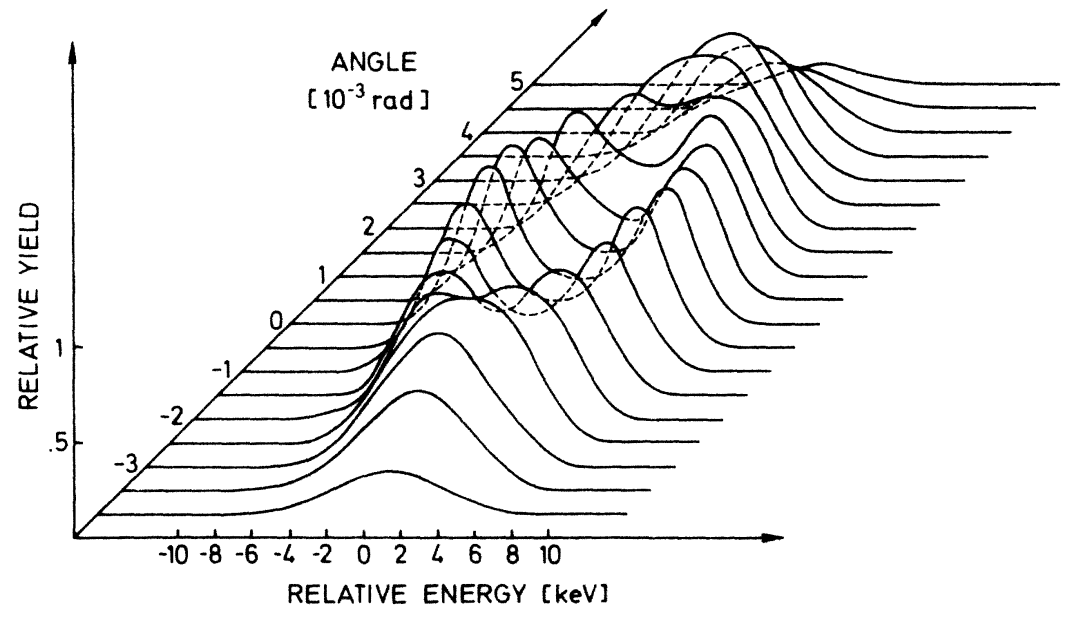

FIG. 3. Joint angleenergy distribution. Energy is measured relative to 1 $\mathrm{MeV}$. 
kinetic energy per proton of

$$
\begin{aligned}
E_{f} & =\frac{1}{2} M_{p}\left(\overrightarrow{\mathrm{v}}_{\mathrm{inc}}+\overrightarrow{\mathrm{v}}_{p}\right)^{2} \\
& =\frac{1}{2} E_{\mathrm{inc}}+\frac{1}{2} E_{p}+\left(E_{\mathrm{inc}} E_{p}\right)^{1 / 2} \cos \theta .
\end{aligned}
$$

Here $\theta$ is the polar angle determined by the molecular orientation relative to the incident beam direction, and $E_{p}$ is the initial potential energy of the repelling protons, i.e.,

$$
E_{p}=M_{p} v_{p}^{2}=e^{2} / r_{12},
$$

where $r_{12}=1.06 \AA$ is the average distance between the protons in $\mathrm{a}_{2}{ }^{+}$molecule. The final polar angle $\phi$ in the laboratory at which the proton will be detected is determined from

$$
\tan \phi=v_{p} \sin \theta /\left(v_{\text {inc }}+v_{p} \cos \theta\right) .
$$

Since in this work $v_{\text {inc }} \gg v_{p}$, we have

$$
\phi=\left(E_{p} / E_{\text {inc }}\right)^{1 / 2} \sin \theta=\phi_{\max } \sin \theta .
$$

Such a small angle approximation is assumed hereafter.

Consider $C\left(\phi, E_{f}, E_{p}\right)$, the normalized joint final angle and energy distribution resulting from an initially uniform spherical distribution for the $\mathrm{H}_{2}{ }^{+}$molecule. Introducing dimensionless variables

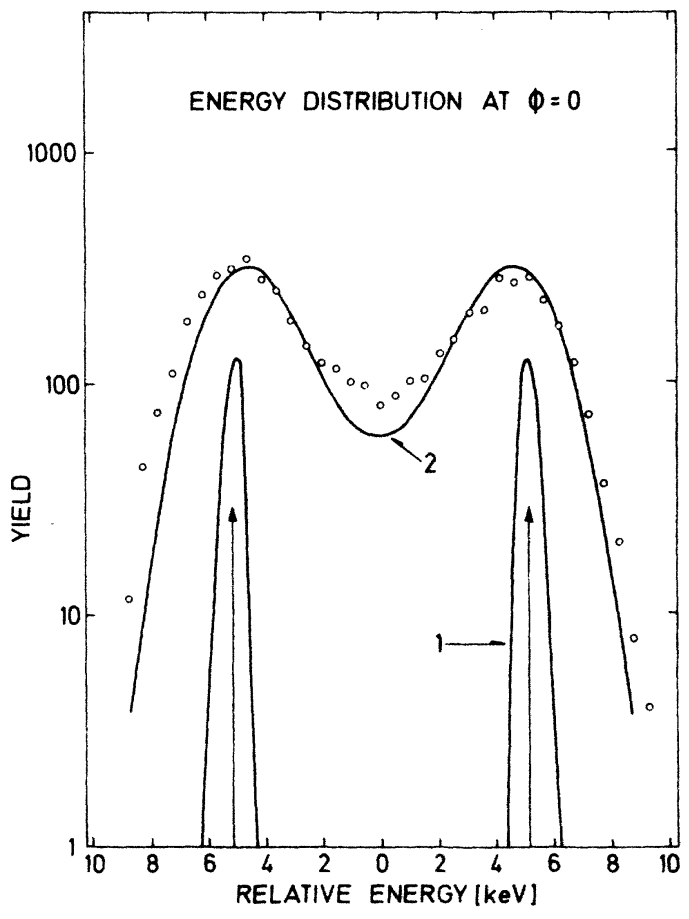

FIG. 4. Comparison of experimental and calculated distributions for $\phi=0 . \bigcirc$ : experimental points; 1 : distribution with zero point motion; 2: calculated distribution taking into account two-component multiple scattering and energy resolution as observed for $1-\mathrm{MeV}$ $\mathrm{H}^{+}$on the same target foil.

$$
\begin{aligned}
& \Phi=\phi / \phi_{\max }, \\
& \xi=\left(E_{f}-\frac{1}{2} E_{\mathrm{inc}}-\frac{1}{2} E_{p}\right) /\left(E_{\mathrm{inc}} E_{p}\right)^{1 / 2}
\end{aligned}
$$

with differential solid angles

$$
d \Omega=\Phi d \Phi d \chi=\phi d \phi d \chi / \phi_{\max }^{2}=d \omega / \phi_{\max }^{2},
$$

we have

$C\left(\phi, E_{f}, E_{p}\right) d \omega d E_{f}$

$$
=d \Omega d \xi \frac{1}{4 \pi \Phi} \int_{-1}^{1} d(\cos \theta) \delta(\Phi-\sin \theta) \delta(\xi-\cos \theta)
$$

if $\Phi \leqslant 1$, while for $\Phi>1, C$ is zero. $\chi$ is the angle measuring the azimuthal orientation of the molecule.

Using $C$, other distribution functions of interest may be easily determined. For example, the integral energy distribution is

$$
\begin{aligned}
A\left(E_{f}, E_{p}\right) d E_{f} \equiv d E_{f} \int d \omega C & =\frac{1}{2} d \xi, & & |\xi| \leqslant 1, \\
& =0, & & |\xi|>1 .
\end{aligned}
$$

Similarly, the integral angular distribution is given by

$$
\begin{aligned}
B\left(\phi, E_{p}\right) d \omega \equiv d \omega \int d E_{f} C & =\frac{1}{2 \pi}\left(1-\Phi^{2}\right)^{-1 / 2} d \Omega, & \Phi \leqslant 1, \\
& =0, & \Phi>1 .
\end{aligned}
$$

In order to represent the effect of multiple scattering in the foil on the final results, consider the "folding" of a Gaussian angular-distribution function with $C$. Thus, let

$D\left(\phi, E_{f}, E_{p}\right) d \omega d E_{f}=d \omega d E_{f} \int d \omega^{1} C\left(\phi^{\prime}, E_{f}, E_{p}\right) M(K)$.

Here

$$
M(K)=\frac{1}{\pi\left(\alpha \phi_{\max }\right)^{2}} \exp \left(-\frac{K^{2}}{\left(\alpha \phi_{\max }\right)^{2}}\right),
$$

and

$$
K^{2}=\phi^{2}-2 \phi \phi^{\prime} \cos \chi^{\prime}+\phi^{\prime 2},
$$

where $\alpha$ measures the dimensionless angular extension of the multiple scattering in the foil. One then finds for each component Gaussian term

$$
\begin{aligned}
D\left(\phi, E_{f}, E_{p}\right) d \omega d E_{f}= & \frac{1}{2 \pi \alpha^{2}} \exp \left(-\frac{\Phi^{2}+1-\xi^{2}}{\alpha^{2}}\right) \\
& \times I_{0}\left(\frac{2 \Phi}{\alpha^{2}}\left(1-\xi^{2}\right)^{1 / 2}\right) d \Omega d \xi, \\
& |\xi| \leqslant 1, \\
& =0, \quad|\xi|>1,
\end{aligned}
$$

where $I_{0}$ is a modified Bessel function. With $D$ one may now fold a Gaussian distribution to account 
for the energy resolution of the particle-detection system, i.e.,

$$
N=\frac{1}{\sqrt{\pi}} \epsilon\left(E_{\mathrm{inc}} E_{p}\right)^{1 / 2} \exp \left(-\frac{\left(E_{f}-E_{f}^{\prime}\right)^{2}}{\epsilon^{2} E_{\mathrm{inc}} E_{\mathrm{p}}}\right),
$$

with $\epsilon$ being a dimensionless measure of the energy resolution. If the argument of the modified Bessel function is quite small $(\Phi \simeq 0)$, this program may be carried to completion analytically, giving

$$
\begin{aligned}
F\left(\phi, E_{f},\right. & \left.E_{p}\right) d \omega d E_{f} \\
= & \frac{1}{4 \pi \alpha\left(\alpha^{2}-\epsilon^{2}\right)^{1 / 2}} \exp \left(+\frac{b^{2}-a c}{a}\right) \\
& \times\left[\operatorname{erf}\left(\frac{b}{\sqrt{a}}+\sqrt{a}\right)-\operatorname{erf}\left(\frac{b}{\sqrt{a}}-\sqrt{a}\right)\right] d \Omega d \xi
\end{aligned}
$$

for $a \neq 0$. Here

$$
a=\frac{1}{\epsilon^{2}}-\frac{1}{\alpha^{2}}, \quad b=-\frac{\xi}{\epsilon^{2}}, \quad c=\frac{\xi^{2}}{\epsilon^{2}} .
$$

For $\Phi \neq 0$ we have resorted to numerical methods to determine $F$. The calculated results shown in
Fig. 4 have been obtained with the above considerations in mind.

For the sake of completeness, the energy angle distribution may be calculated including only the effect of zero-point motion of the incident molecule (assumed to be in a harmonic oscillator ground state). For this purpose the distribution of $E_{p}$ should be calculated and folded with $C$. The result is

$$
\begin{aligned}
H\left(\phi, E_{f}\right) d \omega d E_{f}= & \frac{1}{2 \pi^{3 / 2}}\left(\xi^{2}+\Phi^{2}\right)^{-5 / 2} \\
& \times \Gamma \exp \left(-\Gamma^{2} \frac{1-\left(\xi^{2}+\Phi^{2}\right)}{\xi^{2}+\Phi^{2}}\right) d \Omega d \xi,
\end{aligned}
$$

where

$$
\Gamma=\left(h \omega_{H} \frac{1}{2} M_{p} v_{0}^{2} / E_{p}^{2}\right)^{1 / 2} .
$$

$h \omega_{H}$ is the distance between oscillator energy levels, and $\frac{1}{2} M_{p} v_{0}^{2}$ is the kinetic energy of a proton at the Bohr velocity. This result is also plotted in Fig. 4 for $\Phi=0$.
*Present address: Physics Department, Brookhaven National Laboratory, Upton, New York 11973.

${ }^{1}$ A. D. Bacher, E. A. McClatchie, M. S. Zisman, T. A. Weaver, and T. A. Tombrello, Nucl. Phys. A 181, 453 (1972).

${ }^{2}$ J. M. Caywood, T. A. Tombrello, and T. A. Weaver, Phys. Lett. 37, 350 (1971).

${ }^{3}$ F. H. Eisen and E. Uggerh $\phi$ j, Radiat. Eff. 12, 233 (1972).
${ }^{4}$ J. C. Poizat and J. Remillieux, J. Phys. B $\underline{5}, 94$ (1972). ${ }^{5}$ N. Stolterfoht, F. J. de Heer, and J. Van Eck, Phys. Rev. Lett. 30, 1159 (1973).

${ }^{6}$ E. Laegsgård, F. W. Martin, and W. M. Gibson, IEEE Trans. Nucl. Sci. NS-15, No. 3, 239 (1968).

${ }^{7} \mathrm{G}$. Herzberg, Molecular Spectra and Molecular Structure (Prentice-Hall, New York, 1939). 